\title{
THE POWER OF METHODS FOR THE DETECTION OF MAJOR GENES AFFECTING QUANTITATIVE CHARACTERS
}

\author{
IAN MCMILLAN* and ALAN ROBERTSON \\ Institute of Animal Genetics, University of Edinburgh
}

Received 5.vii.73

\begin{abstract}
SUMmaRY
Current methods for the detection of loci affecting quantitative characters in Drosophila may be in error in two ways,

(i) The detection of loci which do not exist.

(ii) The magnification of the estimated effect of those major loci which do exist by accumulating to their effect those of undetected loci close to them on the chromosome.

This paper considers the magnitude of the second type of error and shows that the estimates of the effect of major loci may be too high by up to five times the standard error of measurement of each cross-over chromosome examined.
\end{abstract}

\section{LOGATION OF GENES}

By modifications of the methods used for the location of visible genes in Drosophila, Thoday and his group have had considerable success in the detection and location of loci affecting quantitative characters, in particular sternopleural bristles (see, for instance, Thoday, Gibson and Spickett, 1964). The results have recently been summarised by Davies (1971). In this present paper, we shall consider some aspects of the discriminatory power of the techniques, which may be illustrated as follows.

Suppose we wish to analyse a chromosome from a line selected upwards for a quantitative character. This may be presumed to have accumulated alleles at loci affecting the character which increase the score of individuals carrying them. In the analysis, we use a tester stock marked with two recessive visible genes and containing no genes in the region between these two markers which have a higher effect on the metric character than the allele present in the chromosome to be tested. This condition is critical and will be examined in more detail later. Chromosomes containing a crossover in the marked region are obtained from heterozygotes between tester and tested chromosome and their effect on the character measured. The measurements may be made in either the heterozygous or homozygous state but, in general, they can be replicated at the will of the experimenter and the mean score obtained to any degree of precision. Suppose there are two loci affecting the character, equally spaced between the markers and one with twice the effect of the other, and that we isolate cross-over chromosomes containing marker $b$. If the standard error of the measurement on an individual chromosome is small relative to the effects of the loci, the distribution of mean scores of separate cross-over chromosomes containing $b$

* Present address; S.I.S. Division, Food and Drugs Directorate, Ottawa, Canada. 
will be as in fig. 2 where each stroke is the mean score of a single chromosome. The chromosomes in the three groups then come respectively from cross-overs in the three regions into which the distance from $A$ to $B$ has been divided. There will, of course, be a mirror image set of chromosomes in which the marker $a$ has been retained. The existence of interactions between the effects of different loci may, however, complicate the correspondence between the two sets.

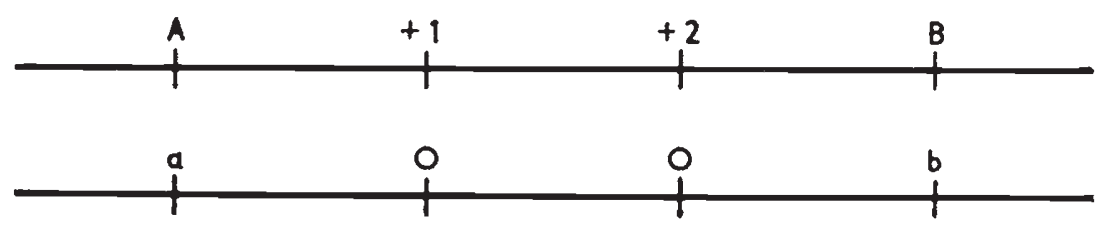

Frg. 1.-An illustration of the technique. The first chromosome contains wild-type alleles at loci $a$ and $b$ and alleles at the two loci affecting the character with affects of +1 and +2 relative to those in the second tester chromosome.

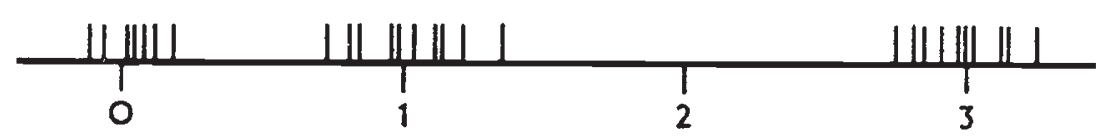

FIG. 2.-A possible distribution of the mean scores of individuals homozygous for separate cross-over chromosomes containing $b$ (see fig. 1) assuming a standard error of measurement of each mean of about $0 \cdot 2$ unit.

We shall then concentrate on the problem of detecting other loci in this region which may affect the character, and in particular on the simplest situation, when there is only one such locus, say between $A$ and the first major locus. The first group of cross-over chromosomes will then be heterogeneous with respect to the mean of the character and this heterogeneity will be greater the closer the locus to the centre of the region and the greater its effect. If the effect is large relative to the error of measurement, the group will clearly split into two. We have then identified a further locus whose position and effect can be estimated from the relative number in the two sub-groups and the difference between them in the mean score for the character.

However, we shall be here mainly concerned with the situation when there is genetic variation in the region which is not detected as heterogeneity. The major locus is then ascribed an effect equal to the difference between the means of the two groups, clearly an overestimate. Note that a further source of bias at the same locus would come from genetic variation in the second region, causing heterogeneity in the second group of cross-overs. In this paper, we are then mainly concerned to answer the question-how large may the effects be which are hidden in groups of chromosomes which are not significantly heterogeneous?

There are several genetic conditions on which the method rests. The first is that all heterogeneity comes from crossing-over between the two markers. This can be satisfied by ensuring that, firstly, both tester and tested are each recent replicates of a single chromosome and, secondly, there is no heterogeneity due to crossing-over in other regions. The latter 
can be controlled either by ensuring that there is no genetic variation between the chromosomes in other regions or that cross-overs in other regions can be detected using further marker loci and excluded. The final and more important assumption is that there is a one-to-one relationship between the order of the cross-over points in the region and the true mean score of individuals carrying chromosomes so derived. For this to hold, at all loci affecting the character the tested chromosome must carry " higher" alleles than does the tester (or vice versa if the tested chromosome is "low"). If this condition is not satisfied and the effects of genes which are the wrong way round are large, then the effects of the separate loci will be wrongly estimated and, perhaps more important, "loci " will be wrongly located.

This may be illustrated by an example in which there are three loci affecting the character in the arbitrary length of 100 units between the two markers as follows:

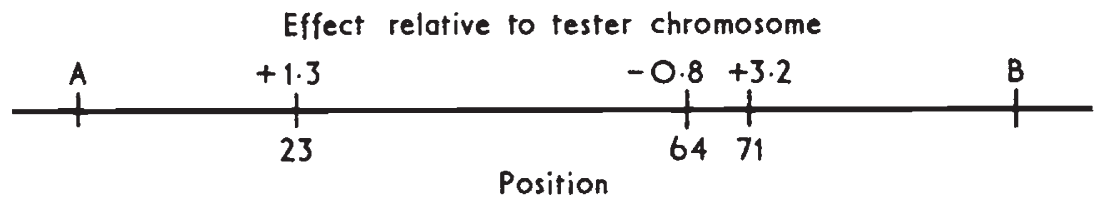

Frc. 3.-A hypothetical example in which the tested chromosome contains both positive and negative alleles, relative to the tester.

Observing cross-overs containing $b$, we then find four groups which in order of the cross-over regions, are given in the first two columns of table 1. Since, in inferring the position of loci, the groups are ordered according to their score for the character, we see that not only are the effects incorrectly estimated but that the locus at 64 is now transposed to 30. It may be very difficult to verify in a particular instance whether this basic condition is satisfied. If a locus " the wrong way round" is close to

TABLE 1

Cross-overs containing $\mathrm{b}$ deriving from a tested chromosome as in fig. 3

$\begin{array}{cccc}\begin{array}{c}\text { Expected } \\ \text { relative } \\ \text { numbers }\end{array} & \begin{array}{c}\text { Mean } \\ \text { values }\end{array} & \text { Position } & \text { Effect } \\ 23 & 0 & 23 & 0.5 \\ 41 & 1.3 & 30 & 0.8 \\ 7 & 0.5 & 71 & 2.4 \\ 29 & 3.7 & - & -\end{array}$

one of the markers, discrepancies will be obvious because cross-over chromosomes will transcend the range of measurement of the parental chromosomes, but otherwise there may be no internal evidence to suggest suspicion. There must certainly be many doubts in situations in which the tester chromosome is merely taken from any stock carrying the necessary marker loci which happens to be available. In the analysis presented by Davies (1971), there are several instances in which he has identified regions with negative effects on otherwise positive chromosomes. These are always located as close to the end of the marked section. If such alleles exist in the middle of a series of 
positive alleles, where they might not be detected, they would vitiate the whole of the analysis; this must cast some doubt on his conclusions. The necessary condition will most likely be met if both tester and tested chromosomes have been selected in opposite directions from the same population, with subsequent backcrossing of the necessary marker genes into the tester. Even so, loci with an effect on the character so small that there is a reasonable probability that the high allele will become fixed in the low tester line, and the low allele in the high tested line, will present limits to the precision of the method which no amount of hard work of measurement or sophistication of design can overcome in subsequent analyses.

\section{THE DETEGTION OF HETEROGENEITY}

We assume that all observed chromosome means are normally distributed about their true value with the same known standard error of measurement. As the null hypothesis is that all $\mathcal{N}$ chromosomes in a group have the same true mean, the obvious test of heterogeneity is a $\chi^{2}$ test (the sum of squares of deviations within the group divided by the square of the standard error of each mean) with $\mathcal{N}-1$ d.f. We can then calculate the power of the test for a given Type I error, i.e. if we accept a chance of 5 per cent falsely claiming heterogeneity, how large must the real effect be at a single locus that the chance of not detecting heterogeneity is less than, say, 10 per cent.

Suppose the locus is at a proportion $p$ of the distance between the marker and the first major locus, that it has an effect $d$ on the character and that $\mathcal{N}$ cross-over chromosomes are in the group. In such samples the number, $m$, of chromosomes which contain the high allele will be binomially distributed with index $\mathcal{N}$ and probability $p$. Given $m$, the $\chi^{2}$ value will be distributed as non-central $\chi^{2}$ with " non-centrality parameter " equal to $m(\mathcal{N}-m) d^{2} n / \mathcal{N} \sigma^{2}$, where $\sigma^{2}$ is the known standard deviation within chromosomes and there are $n$ observations per chromosome (see Scheffé, 1959, p. 412). If, given $m, \mathcal{N}$ and $d$, the probability that the $\chi^{2}$ value will exceed the standard 5 per cent limit for $\mathcal{N}-1$ d.f. is $\theta_{m}$, then the probability, given $p, \mathcal{N}$ and $d$, may be expressed as $\Sigma_{m} \theta_{m}$, where $\pi_{m}$ is the probability of $m$, given $\mathcal{N}$ and $p$. We used the Wilson Hilferty cube-root transformation (see Abramowitz and Stegun, 1965, p. 942) to normalise the non-central $\chi^{2}$ distribution in order to calculate $\theta_{m}$. We then obtained, by interpolation, the necessary value of $d$, given $\mathcal{N}$ and $p$, that the 5 per cent limit will be exceeded on at least 90 per cent of occasions.

Values of $d$ corresponding to this probability of detection of heterogeneity are given in fig. 4. As would be expected, the curves for a given value of $\mathcal{N}$ pass through a minimum when $p$ equals 0.5 , since the expected value of the non-centrality parameter is then highest. As $p$ approaches extreme values, the curves rise asymptotically at a value of $p$, dependent on $\mathcal{N}$, which requires an explanation. However large the effect of the gene, there is no heterogeneity if one of the two chromosomal sub-groups is absent from the sample. Outside this range of $p$, there is a probability greater than 10 per cent that all cross-over chromosomes will have the same true mean. The curves then indicate the two ways in which the locus may be missed; firstly that there is no real heterogeneity amongst the chromosomes in a particular sample, and, secondly, that there is heterogeneity but that this is not detected by the test used. 
The standard error of measurement of each chromosomal mean will depend on the number of replicate measurements made. For a given total amount of measurement labour, we then have to choose between accurate measurement of a few chromosomes or less accurate of many.

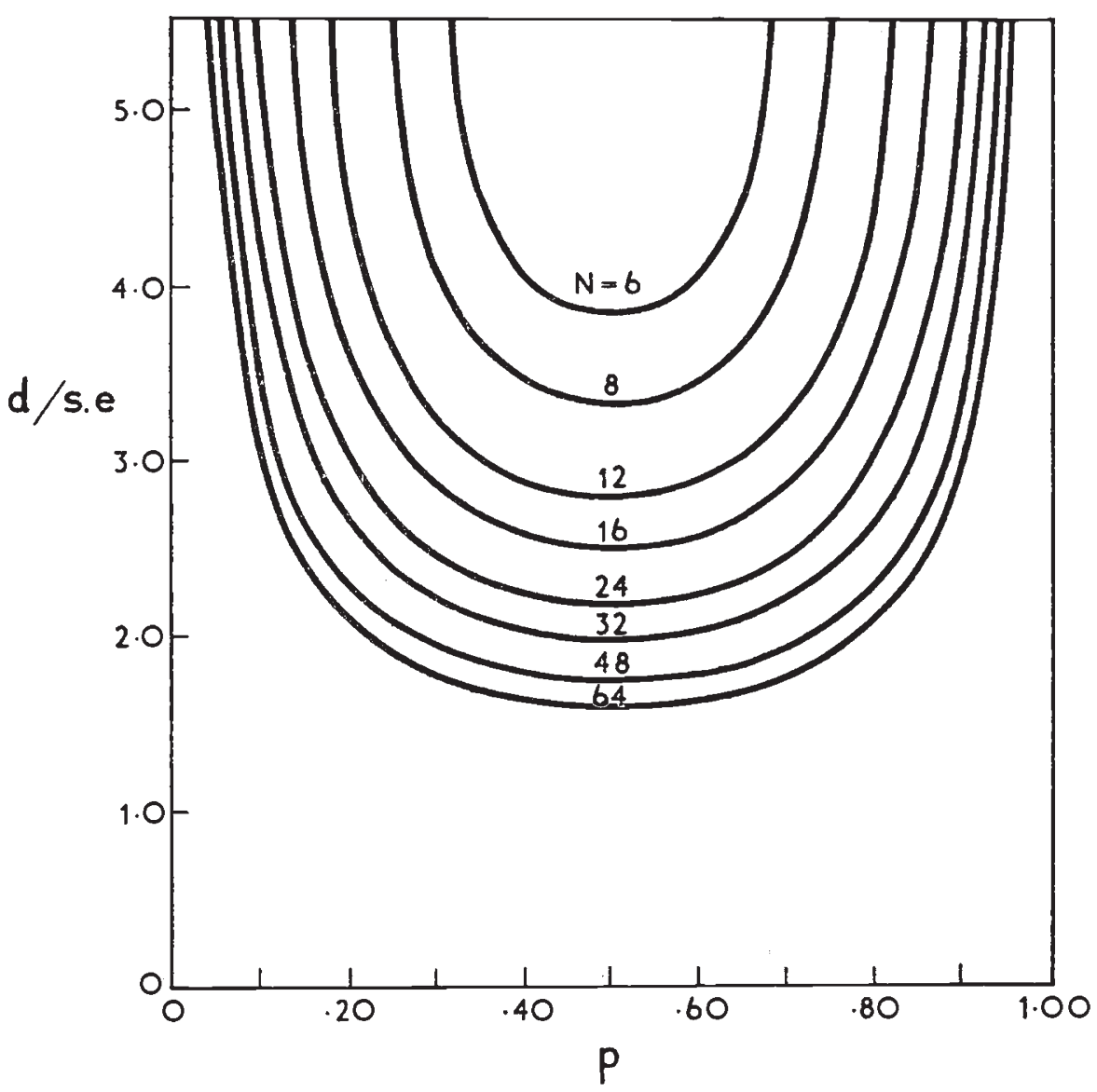

Fig. 4.- The size of effect at a single locus, relative to the standard error of measurement of each chromosomal mean, necessary for a 90 per cent chance of detecting heterogeneity (using a 5 per cent significance level) for various sample sizes and positions of the locus relative to the markers.

Fig. 5 represents the critical value of $\mathrm{d} / \sigma$ as a function of $\mathcal{N}$ and $p$ for 1000 individuals scored over all chromosomes. Each curve, calculated for a specific value of $p$, rises asymptotically at low values of $\mathcal{N}$, corresponding to the probability of homogeneity of the chromosomes in the sample being greater than $0 \cdot 10$, and then passes through a minimum as $\mathcal{N}$ increases. The sensitivity of the method, for a given total amount of measurement, is then highest at an intermediate value of $\mathcal{N}$. But the dependence of this optimum on $p$ is so large as to give little guide in the design of such experiments. 


\section{VARIATION DUE TO MANY SMALI GENES}

We have calculated how large the effect of a single locus in a region has to be before it can be detected as heterogeneity in a group of crossovers. But this is merely one of an infinite number of such models of genetic variation within the region. At the opposite extreme, we might consider a

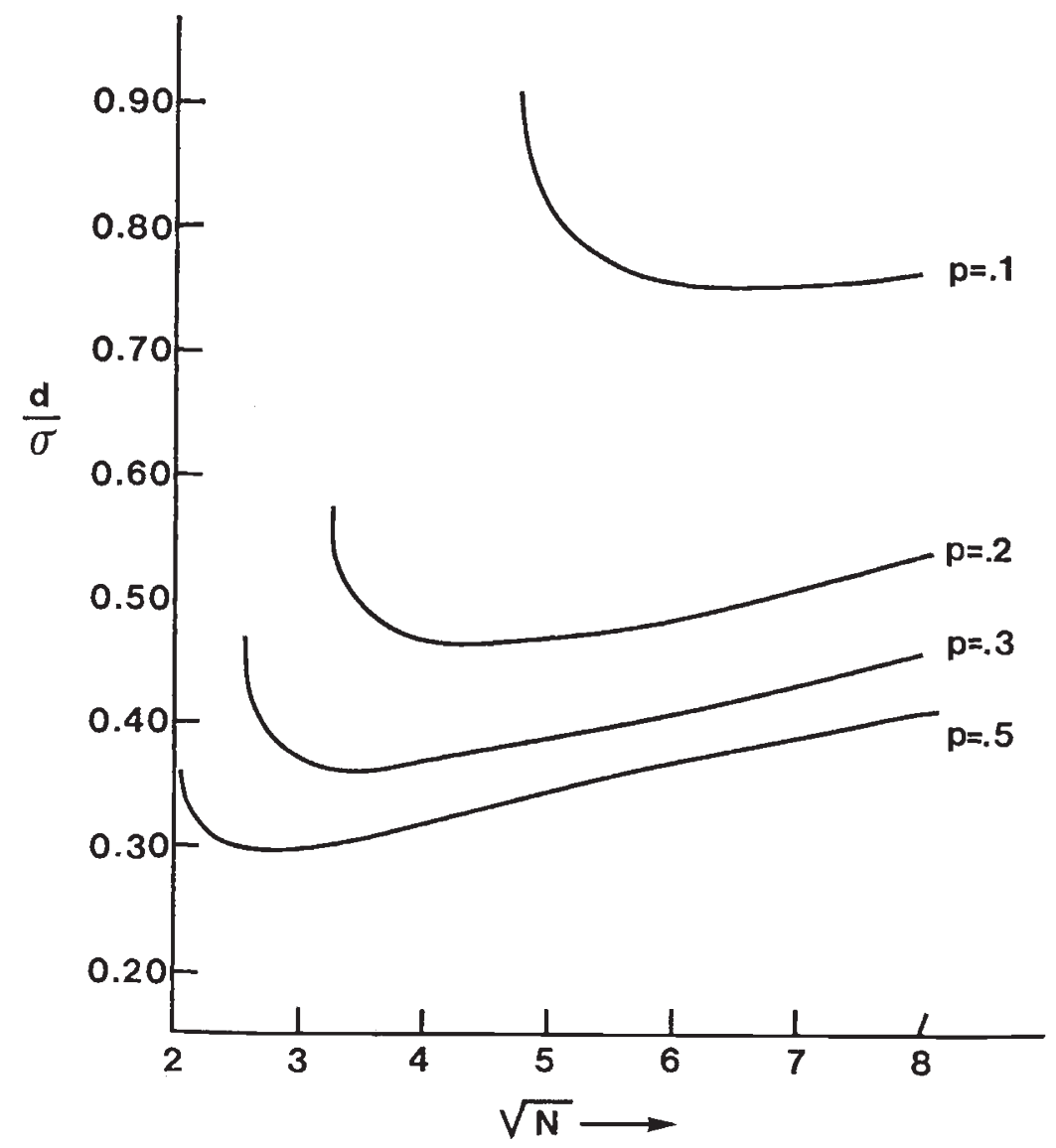

FIG. 5.-The size of effect, expressed relative to the standard deviation of measurement of a single individual, necessary to detect heterogeneity with a chance of at least 90 per cent if 1000 individuals are measured in total.

model in which there are a very large number of loci with small effects distributed evenly over the region. How large then does the sum of these effects have to be before we detect heterogeneity?

In this situation, the distribution of the true effects of cross-over chromosomes is a uniform one with a known range, from which the distribution of observed means is formed by the addition of the error of measurement with a normal distribution. This is not an easy distribution to work with. The problem was then attacked entirely by sampling. We then took, say, eight random observations from a uniform distribution with a given range, added to each a random normal deviate with unit variance and calculated the $\chi^{2}$ value for 7 d.f. for the sample. 
This was repeated 1000 times and the number of samples in which the 5 per cent level of significance was exceeded was noted. The critical value for the range to give a Type II error of 10 per cent was then obtained by trial and error. This proved a very simple and straightforward task for a large computer. The results for sample sizes 8,16 and 32 are given in table 2 .

The values are greater than for a single locus lying close to the middle of the region. This is not unexpected since the variance in the population of possible cross-overs is in that case $d^{2} p(1-p)$ and in the present case is

\section{TABLE 2}

Variation due to many loci with small effects

The total effect necessary to give heterogeneity at the 5 per cent level of significance in 90 per cent of occasions

$\begin{array}{cc}\begin{array}{c}\text { Sample } \\ \text { size }\end{array} & \text { Total effect } \\ 8 & \text { Standard error } \\ 16 & 6 \cdot 30 \pm 0.08 \\ 32 & 4 \cdot 42 \pm 0.06 \\ & 3.60 \pm 0.04\end{array}$

$R^{2} / 12$, where $R$ is the total range of effects. The critical value of $R$ for a given sample size might then be expected to be of the order of $\sqrt{3}$ times the critical value of $d$ when $p=\frac{1}{2}$, which is approximately so.

\section{Discussion}

Apart from the experimental limitations of the method mentioned earlier, one of its drawbacks has been uncertainty about the validity of the conclusions drawn in all but the simplest cases. Almost inevitably, any errors made will be in the direction of ascribing too high a proportion of the total difference between tested and tester stocks in a region to too few loci. A locus is identified as a gap in the distribution, exemplified in fig. 2 . The temptation then is to ascribe this gap to a locus which has an effect equal to the difference between the means of the two groups of chromosomes which bound it. This may be in error for two reasons.

(i) The gap itself may be the consequence of there having been no crossover in a part of the region in which there are several loci affecting the character. There might, for instance, have been two loci with moderate effects very close together between which there had been no cross-overs in the sample. These two would then have been lumped together as a single major gene-perhaps not a major mistake, as at least a concentration of effect has been correctly identified. But the gap may also lump together several small loci as one, if there has been a fairly long stretch of the region with no cross-over. The problems of identification of loci, which will be considered in more detail in later papers, proves to be not so much the detection of a single locus in a region, in which the alternative is no effect at all, but rather to be sure that any major effect discovered is not really the sum of a large number of small ones.

(ii) Any group of chromosomes may conceal heterogeneity due to undetected loci whose effects will then be added to that of the major locus. 
This is the problem with which we have been concerned in this paperhow large are the total effects which may be concealed in groups which we declare to be homogeneous? For a single gene, this clearly depends on its position within the region and the number of cross-over chromosomes in the group concerned. Fig. 4 shows that, if the locus is in the middle of the region with around 10 chromosomes in the group, an effect of less than 3 standard errors of measurement will be missed on more than 10 per cent of occasions. If the region contains a very large number of small effects evenly distributed along it, then the total effect required for the detection of heterogeneity is larger than this and, for a sample of chromosomes, will be in the neighbourhood of 5 times the standard error.

Acknowledgments.--The authors would like to thank Dr W. Hill for reading parts of the manuscript and for helpful suggestions on the presentation of some of the formulae. We are also indebted to Miss Kathy Paver and Miss Jenny Carne for computer assistance.

I. McM. was supported throughout the course of this work by a National Research Council of Canada postdoctoral fellowship.

\section{REFERENCES}

ABRAmowitz, M., AND sTegun, I. A. 1965. Handbook of Mathematical Functions. Dover Publications, New York.

Davies, R. W. 1971. The genetic relationship of two quantitative characters in Drosophila melanogaster. II. Location of the effects. Genetics, 69, 363-375.

THODAY, J. M., GIBSON, J. B., AND SPICKETT, s. G. 1964. Regular responses to selection.

II. Recombination and accelerated response. Genet. Res., Camb., 5, 1-19.

scheffé, H. 1959. The Analysis of Variance. Wiley, New York. 